\title{
Low Bone Mass and Osteoporosis in Women Referring themselves to Dual X-Ray Absorptiometry - Experience with a Health Promotion Action
}

\author{
Gerhard W. Goerres ${ }^{*}, 1$, Jaap Swanenburg ${ }^{2}$ and Daniel Uebelhart ${ }^{2}$ \\ ${ }^{I}$ Institute of Medical Radiology, Buergerspital Solothurn/Spital Grenchen soH, Solothurn, Switzerland \\ ${ }^{2}$ Osteoporosis Center, Department of Rheumatology and Institute of Physical Medicine, University Hospital of Zurich, \\ Zurich, Switzerland
}

\begin{abstract}
Aims: This retrospective analysis was done to describe the difference in the prevalence of osteoporosis/low bone mass between women referring themselves to bone mineral density (BMD) testing with dual X-ray absorptiometry (DXA) and women referred by their family practitioner.

Methods: Women were recruited by a health promotion action in a Swiss weekly periodical and compared with female patients sent by their physician for DXA testing for various medical indications during the same period. Patients under steroid treatment, known previous fracture and undergoing follow-up for low bone mass were excluded. Self referred women were compared to female patients aged 40 years and older and the same evaluation was repeated for women aged 65 and older.

Results: No differences were found in the prevalence of osteoporosis/low bone mass in women referred by their physician $v s$ those who were self referred. However, a significant difference was found with age: the self referred women were 63.1 \pm 8.6 years of age whereas the patient group's mean age was $59.7 \pm 9.4$ ( $\mathrm{p}=0.0001,95 \%$ CI of the difference: $21-61$ years).

Conclusion: We suggest that health promotion actions might be able to recruit the correct candidates for BMD testing, since we found no significant difference in the prevalence of osteoporosis/ low bone mass between self referred and physician referred women. Our data further suggest that physicians may react earlier on their patient's risk profiles than the time frame of action by the self-referred women.
\end{abstract}

Keywords: Osteoporosis, health promotion, dual X-ray absorptiometry.

\section{INTRODUCTION}

Osteoporosis is a growing health care problem in societies with aging populations. Therefore, information of elderly persons to increase awareness of osteoporosis is important. Previous works show that health promotion programs can help to reach populations at risk for osteoporotic fractures, thus, reducing morbidity and mortality [1-3]. Information about osteoporosis and increased fracture risk both for populations as well as individual patients and their treating practitioners is an important part of prevention programs $[4,5]$.

It has been suggested that screening examination of bone mineral density (BMD) using dual energy X-ray absorptiometry (DXA) may improve awareness and impact on initiation of treatment and prevention of osteoporosis [1]. DXA is the standard method for BMD measurements used for the diagnosis of osteoporosis and assessment of fracture risk [6-9]. According to the official positions of the International Society for Clinical Densitometry (ISCD), BMD testing should be considered in women 65 years of age

*Address correspondence to this author at the Institute of Medical Radiology soH, Buergerspital Solothurn/Spital Grenchen, Schoengruenstrasse 42, CH 4500 Solothurn, Switzerland; Tel: +41 (032) 627 4100; Fax: +41 (032) 627 4125; E-mail: ggoerres_so@spital.ktso.ch and older, in postmenopausal women under the age of 65 with risk factors and in men 70 years of age and older [9]. Additionally, patients with diseases or conditions associated with a low bone mass or bone loss and patients taking medication associated with these findings should undergo BMD testing [9]. However, DXA is usually not widely used in screening programs because of its limited availability and relatively high costs.

The aim of this retrospective study was to describe prevalence of osteopenia and osteoporosis in women who referred themselves to BMD testing with DXA as part of a health promotion program. They were compared to female patients who were sent by a family physician. Both the limitations of this health promotion action and the differences between the two groups will be discussed.

\section{MATERIALS AND METHODOLOGY}

\section{Self-Referring Women and Patients}

In 2003 a promotion was launched in a large Swiss weekly periodical entitled 'big osteoporosis action' (Grosse Osteoporose-Aktion der Schweizer Illustrierten). The Schweizer Illustrierte is one of the largest Swiss weekly periodicals in German language with an edition of approximately 250 '000 prints at that time. A voucher was included for the readers of this magazine enabling them to 
have their BMD measured by a standard DXA in one of 19 centres in the German speaking part of Switzerland at a reduced fee (175.00 Swiss Francs). The centres included large district hospitals, specialized orthopaedic/ rheumatology clinics and the three University Hospital centres. Only centres with staff experienced in DXA participated in this promotion.

In the advertisement, a statement was given that BMD measurement should be done regularly in women aged 65 and above. There were no further restrictions indicated on the voucher regarding risk factors of persons who should preferably undergo DXA scanning for the assessment of fracture risk. The promotion was advertised during 4 weeks and the population was free to use the voucher during a 4 months period from September to December 2003). The persons participating in this promotion received the result of their DXA test directly with a hard copy of the measurement results and a diagnosis of normal bone density, osteopenia or osteoporosis. This procedure allowed them to discuss the result to their family physician/ specialist. Furthermore, the voucher included the offer to further discuss the results of the DXA scan with the physician responsible for the measurement. The persons participating in this promotion had to pay their fee directly to the institution and they could not have the DXA and consultation paid by their health insurance.

All the women who took part in this promotion and had DXA scanning at the Osteoporosis Center of the University Hospital of Zurich were included in this evaluation.

Patients sent by their family practitioner with various indications for DXA scanning during the same period served as the reference population for comparison with the population recruited by the health promotion action.

The data were retrospectively evaluated after the end of the promotion period. The evaluation was done in accordance with the ethical guidelines of our institutional review board and the use of data for this retrospective analysis was approved by our local ethics committee.

\section{DXA Measurements}

In our institution, the DXA measurements were performed on the supine patient at the lumbar spine (L2-L4) and the non-dominant hip with a Hologic QDR 4500 A and C device (Hologic Inc., Waltham, MA). The lumbar spine measurement was taken from dorsal projection. The regions of interest (ROI) at the lumbar spine were in the vertebral body L2 - L4. In the hip a total ROI and additional regions of interest were located at Ward's triangle, femoral neck, and trochanter. If measurement was not possible at the lumbar spine or hip an additional measurement of the non-dominant forearm was acquired according to the guidelines of the ISCD [10]. Only the BMD measurement of total spine, total hip and femoral neck region of interest were used for reporting.

BMD was given as absolute values in $\mathrm{g} / \mathrm{cm}^{2}$, and as $\mathrm{Z}$ and, $\mathrm{T}$-score values. The $\mathrm{Z}$-score value corresponds to the number of standard deviations from the mean value that was defined by the range of BMD of an ethnically comparable age and gender-matched reference population. The T-score value corresponds to the number of standard deviations from the mean of a gender-matched reference population of young adults defined as the Peak Bone Mass, as based on data of the Third National Health and Nutrition Examination Survey (NHANES III) provided by the manufacturer. In agreement with the ISCD guidelines, osteopenia or osteoporosis were defined according to the lowest measured value in either spine or hip. Osteopenia, as defined by the WHO classification, corresponds to a T-score value between -1.0 and -2.5 . Osteoporosis was defined a T-score value of -2.5 and lower. Low bone mass is defined as all $\mathrm{T}$-score values indicating osteopenia or osteoporosis [9].

The two DXA devices were cross-calibrated using a spine phantom and daily quality controls using the same phantom were performed during the whole study period, according to the recommendations of the manufacturer. A maximum $1.5 \%$ variability from the mean value is considered as a tolerable variation of the DXA measurements between the two DXA devices and 0.5\% variability for the longitudinal quality control of each individual device. All measurements and quality control were done by the same two experienced technicians during the whole study period. They were experienced with 6 and 9 years at the time of this study and had measured coefficient of variation in short-term precision studies of $1.1 \%$ and $1.2 \%$ for measurements of the lumbar spine and $1.3 \%$ and $1.4 \%$ for measurements of the hip respectively.

\section{Data Analysis}

Age and gender distribution were analyzed for all patients undergoing DXA during the defined period and all participants of the promotion action. To allow for further comparison of the BMD distribution the patients sent by their family practitioners were split into 4 groups according to the indication for DXA scanning and the age distribution of patients was adjusted to the promotion group. Only the females were further analysed. The following 4 groups of indications were created: (i) patients undergoing DXA for a follow-up examination, who already had at least one previous DXA scan in our institution. The patients of group (i) are very likely to have osteopenia or osteoporosis and, therefore, undergo control examinations for treatment monitoring. In group (ii) were patients under documented long term steroid medication for various clinical problems such as rheumatic diseases or patients after organ transplantation. The patients in group (ii) have an exclusively high risk to develop osteopenia and osteoporosis. In group (iii) were patients with documented previous fracture who underwent DXA for the suspicion of osteoporosis related fracture as a first examination before initiation of treatment. The patients in group (iii) are also likely to have a low bone mass, because of the clinical suspicion of an osteoporosis related fracture. In group (iv) all other patients were included sent by their physician with a range of clinical problems such as rheumatologic disease (patients without steroids), endocrine disorders, and suspected malnutrition such as anorexia.

Only patients with adequate quality of DXA measurement were used for the evaluation. For example in patients with bilateral hip prosthesis and degenerative disease at the lumbar spine or osteosynthesis and metal artefacts of the lumbar spine, DXA data was considered not 
to be adequate for this study. For this analysis only results of BMD measurement of the lumbar spine and hip were used. Patients with a diagnosis of normal bone mass, osteopenia, or osteoporosis based on forearm measurements were not included.

\section{Statistics}

The frequencies of osteoporosis and low bone mass at the lumbar spine, femoral neck, and total hip were assessed. The mean value and standard deviation is given for normally distributed values. The ranges of T-score values are tabled since the values can be either negative or positive. Confidence intervals are given at the 95\% level. MannWhitney $U$ test and Fishers' exact test were used for comparison. A p-value of less than 0.05 was considered to be significant and Bonferroni correction was done for multiple measurements when appropriate.

\section{RESULTS}

\section{Patients and Self-Referring Persons}

Six-hundred eighty-eight patients were sent by their family practitioner to undergo DXA scanning between September and December 2003. The data of 29 patients were excluded from this analysis because of insufficient information. Finally, 659 patients (575 females and 84 males, mean age $58.6 \pm 15.3$ years, $14-91$ years) were split into group (i) $n=113$, group (ii) $n=105$, group (iii) $n=114$, and group (iv) $n=327$. For comparison with the self-referring women, only the female patients of group (iv) aged 40 years and older were further analyzed (262 of the 327 patients of group (iv)). The age distribution of these female patients is shown in Fig. (1).

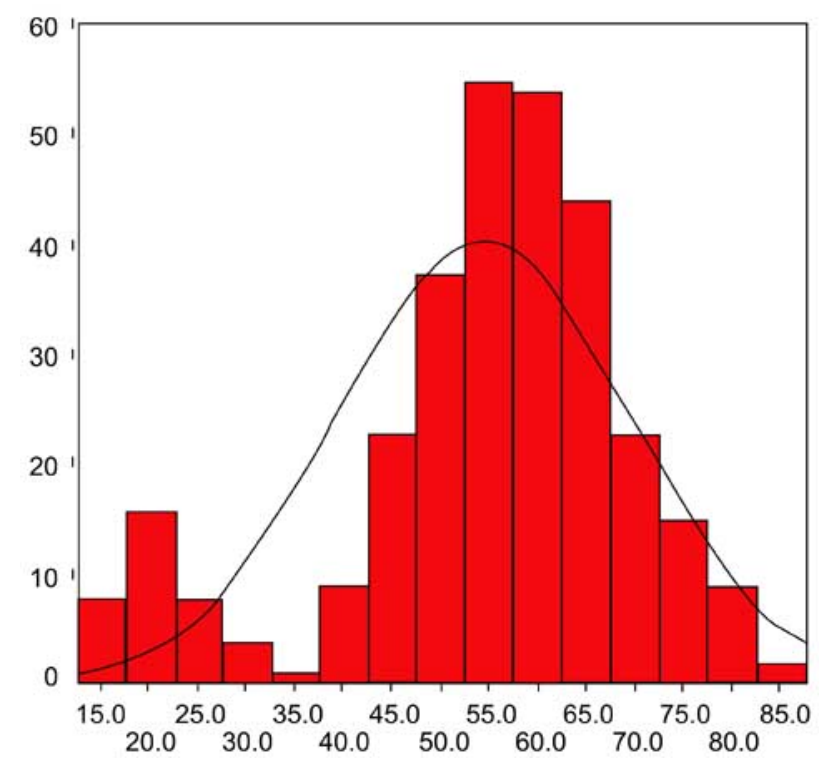

Fig. (1). Age distribution of female patients of group (iv). The female patients of group (iv) who were sent by a family practitioner show a bimodal age distribution, since also young patients with various diseases, such as anorexia, malabsorption or rheumatic diseases have been sent for DXA. For further comparison the female patients of group (iv) being 40 years and older were used ( $n=262$; mean age $59.7 \pm 9.4$ years). The 104 females recruited by the health promotion action were also 40 years and older (Fig. 2).
During the same period 111 persons were recruited by the health promotion action to undergo DXA measurement (mean age $63.3 \pm 8.7$ years, $40-84$ years; 104 females and 7 males). All examinations were technically suitable for diagnosis and further evaluation. For further evaluation, only data of the 104 female self-referring persons were used. The age distribution of the self-referring women is shown in Fig. (2).

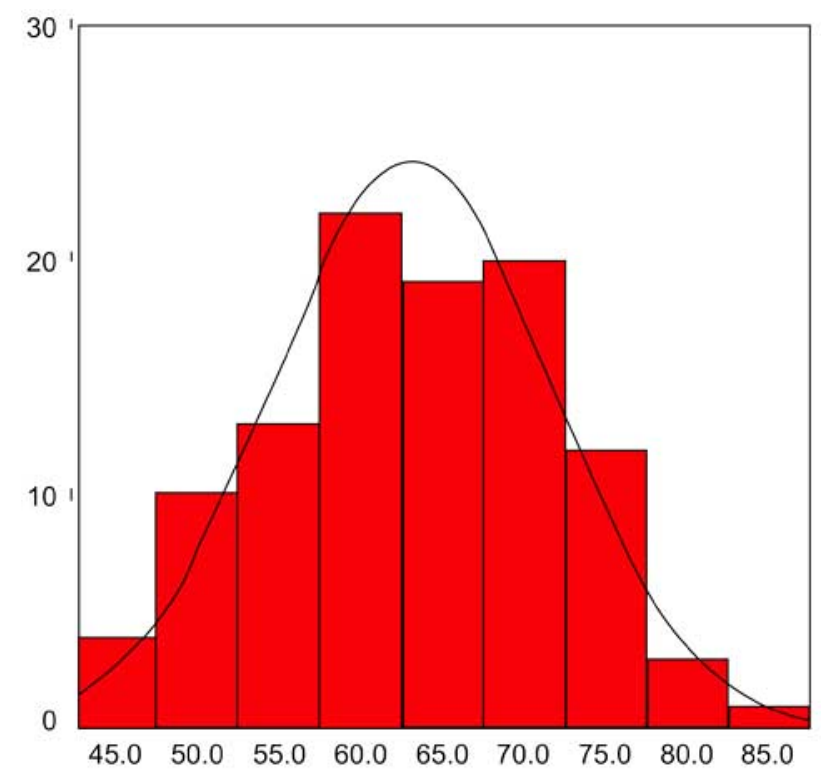

Fig. (2). Age distribution of self-referring women. The 104 selfreferring women had a mean age of $63.1 \pm 8.6$ years.

\section{Comparisons}

We intended to exclude all patients with clear indications for DXA scanning such as given in the groups (i) to (iii) since they were likely to be treated by a family physician and DXA scanning would have probably been paid by insurance companies. Additionally, we excluded the male patients of group (iv) in the final analysis. The female patients of group (iv) were used in order to adapt the patient group send by practitioners to a comparable group of female self-referring persons. The female patients of group (iv), who were referred for scanning by their family practitioner, were used for comparison because they had no long-term steroid medication nor previous fracture and did not undergo followup examination for known bone disease. Hence, the age distribution of patients was adjusted to the self-referring persons and only female patients of 40 years and older were used for further statistical comparison $(n=262)$. There was still a significant difference in average age. The self-referring females had a mean age of $63.1 \pm 8.6$ years while the female patient group was $59.7 \pm 9.4$ years $(p=0.0001,95 \%$ CI of the difference $21-61$ years).

In Table 1 the range, mean and median values and standard deviation of the total T-values of the lumbar spine and the T-values of the femoral neck and total hip are listed. There was no significant difference between the mean $\mathrm{T}$ values of the lumbar spine $(p=0.09)$, total hip $(p=0.06)$, and femoral neck $(p=0.033)$ (significance level after Bonferroni correction was 0.015). The additional evaluation of frequencies of osteoporosis and low bone mass at the lumbar 
Table 1 T-Score Values at the Lumbar Spine, Femoral Neck and Total Hip

\begin{tabular}{|c|c|c|c|}
\hline Self-referring females; range & $-3.900-3.200$ & $-3.500-1.700$ & $-3.000-2.200$ \\
\hline Self-referring females; median value & -1.300 & -1.300 & -0.800 \\
\hline Females of group (iv); range & $-6.400-3.500$ & $-4.100-2.100$ & $-3.400-2.200$ \\
\hline Females of group (iv); median value & -1.300 & -1.300 & -0.800 \\
\hline
\end{tabular}

The range and mean values with standard deviation (SD) of the total T-values of lumbar vertebra L2-4 and the T-values of the femoral neck and the total hip are given for the group of self-referring females and the group of women 40 years and older who had no long-term steroid medication nor previous fracture and who did not undergo follow-up examination for known bone disease. Additionally, the median value is listed showing that the two groups of self-referring females and female patients had similar bone density values.

Table 2 Frequencies of Low Bone Mass and Osteoporosis

\begin{tabular}{|l|c|c|c|c|c|c|}
\hline \multirow{2}{*}{} & \multicolumn{2}{|c|}{ Lumbar Spine } & \multicolumn{2}{c|}{ Femoral Neck } & \multicolumn{2}{|c|}{ Total Hip } \\
\cline { 2 - 7 } & Low Bone Mass & Osteoporosis & Low Bone Mass & Osteoporosis & Low Bone Mass & Osteoporosis \\
\hline \hline Self-referring females $(\mathbf{n}=\mathbf{1 0 4})$ & $62(59.6 \%)$ & $19(18.3 \%)$ & $67(64.4 \%)$ & $7(6.7 \%)$ & $39(37.5 \%)$ & $6(5.8 \%)$ \\
\hline Females of group (iv) $(\mathbf{n}=\mathbf{2 6 2})$ & $161(61.5 \%)$ & $45(17.2 \%)$ & $162(61.8 \%)$ & $30(11.5 \%)$ & $113(43.1 \%)$ & $12(4.6 \%)$ \\
\hline Fisher's Exact p-value & $\mathrm{p}=0.81$ & $\mathrm{p}=0.88$ & $\mathrm{p}=0.72$ & $\mathrm{p}=0.25$ & $\mathrm{p}=0.35$ & $\mathrm{p}=0.60$ \\
\hline
\end{tabular}

The frequencies of osteoporosis and low bone mass, i.e. osteopenia and osteoporosis, at the lumbar spine (T-score value of the lumbar vertebra L2-4), the hip (total hip ROI) and the femoral neck are listed for all females age 40 years and older. Percentage values are given in brackets. Low bone mass is defined as a T-score value indicating osteopenia or osteoporosis, i.e. a value between -1.0 and lower as measured at the lumbar spine and hip. Osteoporosis is defined as a T-score value of -2.5 and lower. After Bonferroni correction for multiple comparisons the significance level is $\mathrm{p}=0.0083$.

spine, femoral neck, and total hip did not reveal a significant difference between the two groups (significance level after Bonferroni correction $\mathrm{p}=0.0083$; Table 2).

Additionally, the same comparison was repeated for women aged 65 to 84 years (self referring females, $n=46$; female patients $n=69$ ) were included. Again, there were no significant differences in the frequency of low bone mass and osteoporosis as measured at the lumbar spine ( $\mathrm{p}$-values 0.67 and 0.29 ), femoral neck (p-values 0.75 and 0.50 ), and total hip (p-values 0.75 and 0.34 ).

\section{DISCUSSION}

In this study no significant differences were observed between the prevalence of low bone mass and osteoporosis between a population of self-referring females participating in a health promotion action and female patients who were sent for various indications for BMD measurement with DXA by a family practitioner. This might indicate that a health promotion action as performed with an advertisement in a weekly periodical can address females with a similar risk profile for osteoporosis as females who are sent for DXA with a clinical indication. In this study the selfreferring persons were approximately 4 years older than the patients sent by their family practitioner, which was a significant difference. This might indicate that the practitioner can react earlier on a patient's risk profile and send the patient to DXA measurement. Thus, for screening purposes, the family practitioner could also have an important impact on the selection of patients.

There are several limitations of this retrospective analysis. The main limitation is certainly the small sample size and the recruitment in only one geographical area
(Zurich, Switzerland) which limits the extension of the results. The reported number is too small to draw meaningful conclusions in the context of screening or health promotion actions. Therefore, such awareness campaigns should be conducted in a controlled setting and should be embedded in a scientific framework. In this health promotion action approximately 250,000 prints of the publication were published yet only 111 subjects self-referred in our DXA centre (one of 19 centres) corresponding to only $0.04 \%$ of the potential population. If in all participating 19 centres as many self-referring women would have been examined, still less than $1 \%$ of the coupons would have been used. This might show that the self-referral campaign was not very successful since the self-referring subjects were only a tiny fraction of the publication circulation. A further limitation is that high risk patients were excluded. If for example a potential self-referring subject is on glucocorticoids perhaps they would not self-refer because of lack of knowledge about fracture risk while the family practitioners would select such a patient for DXA because of their medical knowledge. Therefore, exclusion of higher risk patients might prevent seeing the difference between groups and the value of having a family practitioner.

In the advertisement published in this Swiss weekly periodical no details about risk factors were given. However, a health promotion action will produce the highest impact on a population when optimized to the level of knowledge about the health problem and informing adequately to allow a person to make up one's mind [4,5]. Granted that the practitioners have sent patients at risk for osteoporosis for DXA measurement, we found that the self-referring females 
seen in our setting correctly decided themselves to undergo this screening test.

Furthermore, we interpreted the difference in age distribution in such a way that family practitioners are able to choose the riskier patients for DXA. However, there was no difference in bone density between the groups, a fact that could also indicate that family practitioners in fact are unable to discriminate high risk patients. Another possibility is that the younger population may not read the publication containing the coupons, or a disproportionately small younger population reads the publication relative to the older age group. The latter introduces a selection bias in the strategy of self-recruitment through the coupons.

It remains unclear whether our results are relevant to other settings or patient populations even regarding the same health promotion action and our evaluation sheds light on the difficulties interpreting self referral bias and performing a valuable health promotion action. Since DXA is not an optimal tool for population screening because of its limited availability and relatively high costs, patient selection for BMD testing is essential to increase the pre-test probability of the disease [11].

We suggest that future health promotion actions on BMD testing in Switzerland should be scientifically accompanied to evaluate the public knowledge of the disease, the characteristics of the population screened and the effect on further management of patients identified by the screening program. Additionally, health promotion actions may cause uncertainty in selected populations and, thus, should be conducted in a controlled way [12]. It is unlikely that health promotion actions that are performed every once in a while will have a lasting and measurable effect on morbidity and mortality by a disease. To date, it is not clear if a screening program for early detection of osteoporosis and low bone mass would have an impact on morbidity and mortality in Switzerland and how such a screening program would have to be designed to be cost-efficient and beneficial for the Swiss population.

\section{CONCLUSION}

The results of this retrospective study suggest that effects of health promotion actions are influenced by many factors.
We found no significant difference in the prevalence of low bone mass or osteoporosis between self-referring women and female patients, suggesting that health promotion actions might be able to recruit the correct candidates for BMD testing with DXA. However, we also found that this health promotion action resulted in the recruitment of women being approximately 4 years older than female patients who were sent by their family practitioner. This might indicate an important role for the family physician who did react earlier on the basis of the individual patient's risk profile.

\section{REFERENCES}

[1] Anastasopoulou C, Rude RK. Bone mineral density screening: assessment of influence on prevention and treatment of osteoporosis. Endocr Pract 2002; 8: 199-201.

[2] Curry LC, Hogstel MO. Risk status related to knowledge of osteoporosis in older women. J Women Aging 2001; 13: 71-83.

[3] Akesson K. Principles of bone and joint disease control programs osteoporosis. J Rheumatol Suppl 2003; 67: 21-5.

[4] Spencer SJ. Lack of knowledge of osteoporosis: a multi-centre, observational study. Scott Med J 2007; 52: 13-6.

[5] Lara-Smalling AA, Shelton AJ, Douglas TC, Rianon NJ. Osteoporosis among perimenopausal African-American women: the relationship between sources of information and levels of knowledge about osteoporosis. J Natl Black Nurses Assoc 2004; 15: 40-7.

[6] Kanis JA. Assessment of fracture risk and its application to screening for postmenopausal osteoporosis: synopsis of a WHO report. WHO Study Group. Osteoporos Int 1994; 4: 368-81.

[7] Cummings SR, Black DM, Nevitt MC, et al. Bone density at various sites for prediction of hip fractures. The Study of Osteoporotic Fractures Research Group. Lancet 1993; 341: 72-5.

[8] NIH Consensus Development Panel on Osteoporosis Prevention, Diagnosis, and Therapy. Osteoporosis prevention, diagnosis, and therapy. JAMA 2001; 285: 785-95.

[9] Leib ES, Lewiecki EM, Binkley N, Hamdy RC; International Society for Clinical Densitometry. Official positions of the International Society for Clinical Densitometry. J Clin Densitom 2004; 7: 1-6.

[10] Hans D, Downs RW Jr, Duboeuf F, et al. Skeletal sites for osteoporosis diagnosis: the 2005 ISCD Official Positions. International Society for Clinical Densitometry. J Clin Densitom 2006; 9: 15-21.

[11] Cadarette SM, Jaglal SB, Murray TM, et al. Evaluation of decision rules for referring women for bone densitometry by dual-energy $\mathrm{x}$ ray absorptiometry. JAMA 2001; 286: 57-63.

[12] Hvas L, Reventlow S, Jensen HL, Malterud K. Awareness of risk of osteoporosis may cause uncertainty and worry in menopausal women. Scand J Public Health 2005; 33: 203-7. 